\title{
Inmigrantes y discurso de la inclusión en la política educativa chilena. Reflexiones desde el dilema redistribución o reconocimiento
}

\author{
JORGE ALARCÓN LEIVA* \\ Universidad de Talca (Chile) \\ joalarcon@utalca.cl
}

\begin{abstract}
Resumen
A partir de la evidencia del fenómeno migratorio en Chile, se examina la situación de los estudiantes inmigrantes extranjeros, tomando como referencia la normativa del sistema escolar y la perspectiva del dilema redistribución o reconocimiento. En particular, el texto pretende mostrar las consecuencias de la comprensión generada por dicho dilema en relación con la dialéctica igualdad/diversidad, para explicar los efectos del discurso inclusivo sobre la situación de los estudiantes inmigrantes, considerados como paradigma de grupo minoritario. Con este propósito, se distingue y analiza un particular corpus legislativo que se extiende entre los años 2009-2015, el cual se caracteriza por introducir masivamente la idea de una política educativa inclusiva como ideario a perseguir por el sistema escolar.
\end{abstract}

Palabras clave: inclusión, extranjeros, sistema escolar, reconocimiento, minorías.

\section{Immigrants and discourse of inclusion in educational policy in Chile. Reflections from the redistribution or recognition dilemma}

\begin{abstract}
Based on the evidence of the migration phenomenon in Chile, the situation of foreign immigrant students is examined, taking as a reference the regulations of the school system and the perspective the redistribution or recognition dilemma. In particular, the text aims to show the consequences of the understanding generated by this dilemma in relation to the equality/diversity dialectic, to explain the effects of inclusive discourse on the situation of immigrant students, considered as a minority group paradigm. With this purpose, a particular legislative corpus that extends between the years 2009-2015 is distinguished and analyzed, which is characterized by massively introducing the idea of an inclusive educational policy as ideology to be pursued by the school system.
\end{abstract} Key words: inclusion, foreign, school system, recognition, minorities.

\footnotetext{
* Doctor en Filosofía por la Pontificia Universidad Católica de Chile. Docente e investigador del Instituto de Investigación y Desarrollo Educacional de la Universidad de Talca. Entre sus publicaciones recientes: “¿Nuevo paradigma en la política educativa chilena? Debates sobre educación y transformación social" (2018), "Modernidad, tolerancia, migración" (2018), "Sistema de Aseguramiento de Calidad de la Educación Superior en Chile y la Gestión Institucional por Resultados: análisis, perspectivas y recomendaciones" (2018).
} 


\section{LA CUESTIÓN MIGRATORIA}

Los movimientos migratorios constituyen una de las características de las sociedades modernas (Bauman, 2005). De hecho, se estima que el 3,2\% de la población mundial vive hoy fuera de su país de origen, lo que equivale a cerca de 214 millones de personas (OIM, 2018). Desde esta perspectiva, la significación de la migración de personas y de grupos se ha convertido en un factor relevante para comprender las transformaciones culturales de diversos lugares y contextos (Cierani, García \& Gómez, 2014).

Pavez observa que, conforme a datos de 2016, en "América Latina y el Caribe existen aproximadamente 7,6 millones de personas migrantes, de las cuales $62,8 \%$ representa flujos migratorios entre países de la región" (2017: 99). Asimismo, sostiene que "el promedio regional de niñas y niños nacidos en un país distinto al de residencia es de 10,5\% y no existe un país cuyo porcentaje supere el 30\%" (2017: 99). Por cierto, tal proporción de población infantil corresponde a niños y niñas que participan en flujos migratorios familiares y que demanda - entre otros aspectos básicosatención de servicios educativos en los países de destino.

Chile no es la excepción a esta regla general de incremento de población inmigrante extranjera. En efecto, desde mediados de la década del 90 - y con mayor fuerza a partir del año 2000—, el país se ha consolidado como polo de atracción para personas que, en su gran mayoría, provienen de naciones de la región sudamericana; particularmente de Perú, Argentina, Bolivia y, recientemente, de Colombia. De hecho, estos son los colectivos de inmigrantes más numerosos en el país, representando en conjunto cerca de $63 \%$ del total de extranjeros/as en Chile (INFORME OBIMID, 2016). El incremento en la migración sur-sur y, especialmente, con destino a Chile, se debe, según Galaz, Poblete y Frías (2017) al crecimiento económico alcanzado por el país durante la década de los 90, el que promedió tasas del 7\% anual, lo que ha estado asociado a mayor estabilidad económica y también socio-política ${ }^{1}$.

De acuerdo con el último Informe del Instituto Nacional de Estadísticas de Chile, del 2018, en concordancia con la información recabada mediante el CENSO 2017, la cantidad de inmigrantes internacionales, según período de llegada al país, ha aumentado en un $60,2 \%$ desde antes de los 90 hasta el período de 2010-2017. En el CENSO de 2002, 1,27\% de la

1 Especialmente después de los eventos de octubre de 2018 en Chile, de amplia resonancia internacional, esta afirmación se vuelve controversial. En verdad, no hay razón alguna para pensar que exista una línea continua que permita decir que la estabilidad económica implica estabilidad socio-política. De hecho, hay quienes piensan que, para el caso de Chile, la estabilidad socio-política es, en verdad, silencio y resignación ante la enorme desigualdad generada por políticas de índole neoliberal. 
población residente era inmigrante; quince años después, en abril de 2017, se censó a 746.465 inmigrantes, lo que representa 4,35\% del total de la población residente en el país (con información declarada en lugar de nacimiento).

En cuanto a cantidad bruta de inmigrantes y su distribución territorial en Chile, la Región Metropolitana se ha venido transformando en los últimos años en el lugar preferido para radicarse. En términos de porcentajes, sin embargo, en Santiago los inmigrantes representan un 7\% del total de la población, ubicándose en el cuarto lugar, mientras que en la región de Arica y Parinacota corresponden a un 13,7\% de la población, siendo ésta la región con mayor proporción de inmigrantes con relación a población total.

Ante esta situación, no sólo los migrantes enfrentan nuevos desafíos al verse en un país con costumbres, cultura e incluso, como ocurre con los haitianos, un idioma diferente, sino que, además, el Estado chileno se ve exigido para cumplir con los derechos de las personas que ingresan al país. En este sentido, se plantea un requerimiento recíproco de demandas por derechos por parte de los inmigrantes y la necesidad de proveer sus requerimientos por parte del Estado. Entre esos derechos, la educación es una de las garantías fundamentales a las que debe tener acceso toda persona y es, por tanto, deber de cada nación proveerla a todos y cada uno de sus habitantes, sean estos nacidos dentro o fuera del país. Sin embargo, esta situación de apariencia simple no siempre se concibe e implementa en consecuencia.

En la legislación chilena, el Artículo $4^{\text {to }}$ de la Ley $N^{o}$ 20.370/2009, General de Educación, establece que el Estado otorgará educación obligatoria a todos los estudiantes en los niveles de enseñanza básica y media, que asegurará su acceso y permanencia, proveerá educación gratuita y de calidad, promoviendo la inclusión social y la equidad, así como que velará por la igualdad de oportunidades y la inclusión educativa. Contra esta declaración, no obstante, existe evidencia de las varias dificultades presentadas por el sistema escolar chileno, en cuanto a equidad y calidad, dificultades que se expresan también -y que con frecuencia se expresan de maneras más extremas- en relación con el derecho a educación de los inmigrantes.

La creciente presencia de inmigrantes pone de manifiesto también la necesidad de hacer frente a cada vez mayores niveles de diversidad, provocados justamente por el incremento de la matrícula de estudiantes inmigrantes. En efecto, conforme a datos del Centro de Estudios del Ministerio de Educación de Chile, en 2015 estaban matriculados 30.625 estudiantes inmigrantes, cantidad que se duplicó al 2016, alcanzando un total de 61.086 y creció en 2017, ascendiendo a una cifra de 77.608, mientras que 
en abril de 2018 alcanzaría un 68, 3\%, con un total de 113.585 estudiantes (MINEDUC, 2018a). La diversidad constituye ciertamente un desafío para un sistema educativo acusado con frecuencia de ser, justamente, un sistema homogéneo, sin sensibilidad a las diferencias de contexto de las familias y estudiantes, así como con escasa capacidad de respuesta frente a los diferentes ritmos de aprendizaje. Por mencionar solo algunos de los aspectos en los que la diversidad de revela una dificultad.

Con este panorama como referencia general, el artículo se propone caracterizar, en primer lugar, el dilema entre redistribución o reconocimiento. El dilema en cuestión permitirá ver el conflicto entre una orientación por la igualdad, basada en la identidad de sujetos y grupos, frente a una orientación por el reconocimiento, basada en las diferencias entre individuos y grupos. Enseguida, el texto se aboca a describir brevemente la trayectoria de la política educativa chilena entre 2009 y 2015, haciendo hincapié en la forma en que dichas políticas han jugado un rol preponderante como medio de expresión del discurso inclusivo. Finalmente, se discuten los resultados contradictorios de una política educativa comprometida con la inclusión, pero construida sobre la base de diferencias puramente materiales entre los individuos.

La hipótesis general del texto consiste en sostener que mientras que la orientación de las iniciativas de política educativa ha estado dominada por un discurso inclusivo, los instrumentos que se ha dispuesto para su implementación buscan corregir deficiencias distributivas de la riqueza socio-económica. Así, mientras que el ideario de la política educativa radica en un horizonte valórico, de orden cultural y, en consecuencia, debiera estar orientado por una política de reconocimiento, su realización práctica pretende satisfacerse mediante la introducción de correctores socio-económicos y, en consecuencia, de carácter redistributivo. Así, resulta inevitable la evaluación de que la política educativa no alcanza plenamente su propósito, inscribiéndose en el marco de una forma de "optimismo cruel": el tipo de relación que surge cuando algo que se desea es, en verdad, un obstáculo para conseguirlo (Berlant, 2011: 24).

\section{LA GRIETA ENTRE EL IDEARIO Y SU IMPLEMENTACIÓN}

La problemática planteada hacia el final del primer apartado del artículo, se relaciona con el hecho de que la inclusión, en un lapso que no supera los diez años, se ha posicionado en el centro del discurso educativo chileno (UNESCO, 2015; Armijo-Cabrera, 2018), tanto en el ámbito de la educación especial o diferenciada, i. e. en el ámbito de los servicios educativos orientados a apoyar el desarrollo de personas con necesidades edu- 
cativas especiales (NEE), como en la totalidad del sistema educativo. Podría decirse que en el lapso de tiempo señalado, la inclusión se transformó en el ideario de toda una generación de políticas educativas, al punto que buena parte de las iniciativas se interpreta en relación con su capacidad para abarcar el objetivo comprendido por tal ideario.

Conviene describir cómo ha llegado a ocurrir tal cosa, en un contexto nacional en que la agenda no ha estado precisamente centrada en la inclusión, sino que en calidad, eficiencia y equidad. En alguna medida, la aparición del concepto de "inclusión" obedece a la necesidad de subsumir la orientación de la política en un ideario compartido, de fácil divulgación y de comprensión pública inmediata: la inclusión ha cumplido, en efecto, el rol de servir de pivote para la serie de cambios que siguen teniendo lugar en la política educativa chilena, dotando a la política de sentido y proveyendo una herramienta retórica difícil de contrarrestar.

Para el caso del sistema educativo en su conjunto, la centralidad de la inclusión se verifica, por una parte, en la promulgación de la Ley $\mathrm{N}^{\circ}$ 20.370/2009, General de Educación (MINEDUC, 2009a), y de la Ley N $^{\circ}$ 20.845/2015 (MINEDUC, 2015a), de Inclusión Escolar. En cambio, de forma temporalmente simultánea, para el caso de la educación especial y diferenciada, el posicionamiento de la inclusión en la política se corresponde con la promulgación del Decreto $N^{\circ}$ 170/2009 (MINEDUC, 2009b) y del Decreto N 83/2015 (MINEDUC, 2015b).

Mediante la promulgación de ambos pares de normas se ha pretendido responder, por una parte, al reconocimiento de una mayor diversidad o heterogeneidad del estudiantado matriculado en el sistema escolar; mientras que, por otra parte, pero al mismo tiempo, se hace frente a la exigencia de igualdad de trato. Se trata de normas legales que se enfrentan a la exigencia de tener que ser sensible a la diversidad y a la exigencia de tener que responder a la igualdad. La noción de "inclusión", en efecto, introduce en las normas indicadas un giro que parece liberar a la política educativa de tener que justificar la racionalidad instrumental de su operación, puesto que ella - la inclusión- actúa como un desiderátum.

La comprensión de la inclusión en estas normas legales es, en lo sustancial, expresión del modo cómo la educación chilena considera a minorías en el sistema escolar y por extensión son las normas sobre cuya base se atiende a la diversidad, cualquiera sea su origen, a saber, socioeconómico, sexual, racial o étnico. Lo cual, por cierto, incluye a los inmigrantes extranjeros. De hecho, debe hacerse notar que la palabra "inclusión" presenta 74 ocurrencias en el texto Política Nacional de Estudiantes Extranjeros 2018-2019 (MINEDUC, 2018b), siendo utilizado por cierto en distintas fórmulas. Más importante que esto, no obstante, el texto que sinte- 
tiza el conjunto de disposiciones relacionadas con la situación de estudiantes extranjeros se encuentra presidido por iniciativas redistributivas y de reconocimiento. Estas iniciativas repiten el patrón que se pretende subrayar en el presente trabajo: la política educativa sobre estudiantes extranjeros es derivada de la política educativa sobre minorías.

Como puede constatarse, las normas referidas han sido promulgadas entre 2009 y 2015, y algunos de sus elementos suscita la posibilidad de considerarlas vinculadas. En efecto, el año 2009 se publica la Ley $\mathrm{N}^{\circ}$ 20.370 (MINEDUC, 2009a), General de Educación, en el marco de la cual se establece una nueva definición legal para la educación especial $-\mathrm{y}$, consecuentemente, de la atención a personas con NEE-. El mismo año 2009, se establecen las condiciones técnico-pedagógicas del diagnóstico y de los apoyos requeridos por personas que presentan NEE, mediante la promulgación del Decreto No 170 (MINEDUC, 2009b). Tanto la Ley como el Decreto, por tanto, contienen sendas expresiones referidas a la forma en que la diversidad ha de ser tratada, elevando a la educación especial y diferenciada al estatus de "Modalidad Educativa" y definiendo los recursos técnicos-pedagógicos y financieros para su implementación. Todo ello bajo el rótulo de la "inclusión".

Por otra parte, se ha aludido a la promulgación del Decreto $\mathrm{N}^{\circ}$ 83/2015 (MINEDUC, 2015b) sobre diversificación de la enseñanza y, el mismo año, a la aprobación de la llamada "Ley de Inclusión" (Ley $\mathrm{N}^{\mathrm{o}}$ 20.845/2015) (MINEDUC, 2015a). Es interesante que se indique en la última que se trata de una ley "de inclusión", en circunstancias que su contenido se refiere a la selección escolar, el copago o financiamiento compartido y a la regulación de las ganancias de los propietarios de los establecimientos escolares particulares subvencionados. Vale decir, en ningún aspecto de la ley se establece una política de "inclusión" escolar sensu stricto. Salvo que forzosamente se entienda por tal al aspecto de la legislación que se refiere a la selección de estudiantes para el acceso al sistema escolar, la cual queda regulada de manera que evita los criterios discriminatorios aplicados por los propietarios - por ejemplo, el ingreso familiar, la condición de los cónyuges o las convicciones religiosas del padre y/o madre ${ }^{2}-$.

Otro tanto puede decirse del Decreto No 83/2015, mediante el cual se "[a]prueba criterios y orientaciones de adecuación curricular para estudiantes con necesidades educativas especiales de educación parvularia (sic)

2 Que la llamada "Ley de inclusión" no se refiera a "inclusión", lo revela el que la discusión sobre sus efectos se extendiera hasta comienzos de 2019, en virtud de la réplica a la iniciativa original propuesta en 2018 por el Ejecutivo, mediante el proyecto de ley llamado de "Admisión Justa" (finalmente desechado en el Congreso Nacional). Que se hablara en esta réplica de "Admisión" para referirse a la selección escolar, pone de manifiesto que su cuestión es el acceso al sistema escolar, no la inclusión sensu stricto. 
y educación básica" y se introduce el Diseño Universal de Aprendizaje (DUA) "[...] cuyos criterios buscan promover prácticas inclusivas constituyendo el primer paso para responder a las diferencias individuales en el aprendizaje que presentan los estudiantes" (MINEDUC, 2015b: 17). En esta norma, como puede verse, se trata de promover "prácticas inclusivas", mediante adecuaciones curriculares decididas en virtud de "principios que orientan" el DUA (MINEDUC, 2015b: 20ss), un conjunto definido de herramientas pedagógicas proporcionadas a través de la atención a la diversidad. En esta lógica, importa subrayar que la diversidad se traduce como la diferencia de algunos/as estudiantes, que han sido diagnosticados como habiendo presentado NEE y, en consecuencia, se demanda inclusión para un grupo de estudiantes.

Como puede percibirse, se trata de una singular sincronía legislativa, que permite constatar la simultánea consideración de la inclusión como un propósito general del sistema educativo y como un modo específico de implementar la atención pedagógica frente a la diversidad, diagnosticada por medio de instrumentos técnicos como NEE. Dicho de esta manera, parece referirse a la inclusión en dos esferas distintas, desconectadas entre sí.

Sin embargo, la desconexión es aparente, puesto que la política educativa revela que la inclusión es considerada una manera de comprender la atención a la diversidad y también como el modo de abordar educativamente a múltiples grupos "focalizados", "minoritarios", "subordinados" o "marginados", tanto por condiciones culturales, como sociales y económicas (Ainscow \& Miles, 2008; Graham \& Slee, 2008; Rambla et al., 2008). En ambos casos, nótese bien, se trata de una inclusión pensada para grupos o colectivos que bien pueden servir de base para extender la inclusión a otros grupos o colectivos, inmigrantes, desde luego, pero en ningún caso se la entiende como abierta a todos.

Conforme a las normas referidas la inclusión se constituye, en consecuencia, en un ideario general perseguido por el sistema escolar, que se expresa en la exigencia de atención a la diversidad, pero siempre y necesariamente circunscrita a grupos (diversos), en relación con los cuales se definen mecanismos adicionales de financiamiento. Surge entonces el sentido de la utilización de la palabra "inclusión". No sólo -aunque sí primariamente- en las normas legales, puesto que estas dan forma jurídica a una comprensión primaria de la educación. Se entiende así que las normas legales son la manifestación "positiva" (jurídicamente hablando) de un cierto conjunto de valores compartidos por la comunidad política de la que es expresión y, por lo tanto, cuando se habla de "inclusión" los cuerpos legales manifiestan una orientación de reconocimiento a todos por parte del sistema educativo escolar. "Todos", no obstante, considerados 
en relación con múltiples grupos "focalizados", "minoritarios", "subordinados" o "marginados".

Pero ello no sólo ocurre en Chile. En efecto, la inclusión se ha convertido tanto en una consigna educativa - la llamada "educación inclusiva" o "inclusión educativa" o "inclusión escolar" (Rambla et al., 2008)-, cuanto en una bandera de justicia social - la llamada "inclusión social" (Graham \& Slee, 2008; Knight, 1999; Linblad \& Popkewitz, 2001; Pelletier, 2011). La amplitud de la preeminencia de la inclusión, pudiera entenderse o bien como un síntoma de la medida en que el sistema educativo intensifica su capacidad para generar exclusiones, guiado por criterios cuya acumulación sobre los mismos sujetos ha sido mostrada por varios estudios (Tomlinson, 1982; Madero, 2011; Rothon, 2007, 2008), o bien como el resultado de la natural evolución de la política educativa, que tendería a hacer más efectivos sus instrumentos para mejorar las trayectorias escolares de un mayor número de grupos, como si se tratara de un proceso de perfeccionamiento sin fin (Booth \& Ainscow, 2002; Ainscow \& Miles, 2008; Blanco, 2006; Armijo-Cabrera, 2018). Es decir, se daría mayor énfasis a la inclusión en aquellos contextos en que la exclusión se intensifica y también habría más inclusión en la medida que la política educativa progresa en su capacidad para responder a los requerimientos sociales.

Cualquiera que se juzgue que es la razón de ser de su aparición en la escena pública, el balance respecto de los efectos de las políticas educativas inclusivamente motivadas es, por lo común, poco alentador. En este sentido, Ahmed (2007) ha introducido la idea de "fatiga de equidad", para indicar la transformación del lenguaje de la diversidad en una vacía aspiración institucional, sugiriendo que se ha tratado más bien de una estrategia política de neutralización y control sobre minorías excluidas que de una acción política con impactos determinables. Una comprensión que ha encontrado eco en Chile, en los análisis, entre otros, de Infante y Matus (2009; Matus \& Infante, 2011): las llamadas "políticas inclusivas" no son más que instrumentos de normalización de la situación de grupos minoritarios.

En este último caso, se ha pretendido entender el ímpetu de la noción de "inclusión" en la política educativa nacional chilena, como consecuencia de la alineación de las políticas educativas con las tendencias fomentadas por organismos multilaterales, ordenadas por la "globalización neoliberal". En cuanto a su contenido específico, tal alineación ideológica habría dado lugar a una invisibilización de los mecanismos de "normalización", arrojando luz, paradójicamente, sobre las "diversidades" (Matus \& Infante, 2011), en la medida en que la profundización de patrones de homogeneización institucional han terminado por hacer evidente la necesi- 
dad de una mayor sensibilidad de sentido opuesto, es decir, hacia la heterogeneidad. Esta dialéctica entre un sentido y otro, con todo, sería el resultado de un movimiento conducente a la recíproca anulación del potencial cultural de las diversidades y del poder político de la demanda por igualdad, dando lugar con ello a una tendencia hacia la regulación y el control: una tecnología social, en el sentido de Foucault.

\section{LA INCLUSIÓN ENTRE REDISTRIBUCIÓN Y RECONOCIMIENTO}

Al caracterizar la coherencia semántica y la justificación política de los usos de la noción de "inclusión" en el sistema escolar, en contextos discursivos cuyas diferencias se ponen de manifiesto tanto por lo que concierne a las dimensiones cuantitativas de los subsistemas en que se aplica - el aula, el colegio, el sistema educativo, la sociedad-, como a cuestiones científicas propias de las disciplinas académicas y de la teoría política, se hace relativamente evidente la presencia de grietas de coherencia y de justificación. Este ha sido parte importante del propósito del apartado precedente.

Ahora bien, se pretende no solo constatar que tales grietas existen, sino que se requiere avanzar en busca de una explicación que vaya más allá de su sola descripción. En este último sentido, se propone considerar los usos del concepto de "inclusión", en relación con las teorías de la identidad y del reconocimiento (Taylor, 1996; Hönneth, 1992, 2006), por una parte, y la noción correlativa de justicia social, por otra (Fraser, 1997; Fraser \& Hönneth, 2003).

La "idea de justicia" (Sen, 2009) sobrevuela los desafíos educativos en Chile en diversos contextos y en razón de distintos aspectos de su desenvolvimiento efectivo. La idea de justicia, no obstante, suele aparecer como un referente relativamente menos desarrollado que otros, entre los asuntos que atraen la atención de los investigadores en educación (un caso distinto es Walzer (1997) y subordinada a otras cuestiones, Gutman (1987), y Hönneth, 2013)). En efecto, más comúnmente los problemas de la justicia en el tratamiento de los problemas educativos, se consideran devaluadamente como resultado de la segregación, la discriminación y el elitismo que, por cierto, están relacionados con la justicia, pero sin constituir en sí mismos un problema de justicia ${ }^{3}$.

\footnotetext{
3 Esta afirmación es quizás excesiva, por lo que vale la pena una explicación acerca de su pretensión. Por supuesto que hay trabajos sobre justicia en educación, piénsese en Tomasevsky (2004) cuyos temas despliegan de diversos modos la pregunta por la justicia, pero nunca llegan a tratar directamente la cuestión, como sí lo hacen Walzer y Gutman. Estos últimos, por otra parte, plantean la cuestión desde una perspectiva "normativa", es
} 
En este trabajo se utiliza una idea de justicia como referente para evaluar la coherencia de los diversos usos de la inclusión y se considera que solo una teoría normativa de la justicia hará posible salir de la incómoda situación en que se percibe estando al ideario de la inclusión.

Este propósito se inscribe en el núcleo de lo que Fraser (1997: 20) llamó el "dilema redistribución-reconocimiento"; en virtud de cuya existencia, Fraser ordena, en primer lugar, aquello que constituiría la base fundamental de la pretensión de las exigencias redistributivas y de reconocimiento. Dice:

Las exigencias de reconocimiento asumen a menudo la forma de un llamado de atención a la especificidad putativa de algún grupo, cuando no la crean efectivamente mediante su actuación y, luego, la afirmación de su valor. Por esta razón, tienden a promover la diferenciación de los grupos. Las exigencias de redistribución, por el contrario, abogan con frecuencia por la abolición de los arreglos económicos que sirven de soporte a la especificidad de los grupos. (Un ejemplo de ello sería la exigencia feminista de abolir la división del trabajo según el género.) Por lo tanto, tienden a promover la desdiferenciación de los grupos. (Fraser, 1997: 24)

Enseguida, Fraser subraya que el resultado de pensar de este modo las exigencias de la justicia redistributiva y de reconocimiento, implica "que las políticas de reconocimiento y las de redistribución parecieran a menudo tener objetivos contradictorios". En efecto, y esto es lo importante aquí, "[m]ientras que las primeras tienden a promover la diferenciación de los grupos, las segundas tienden a socavarla" (Fraser, 1997: 25).

De forma que los dos tipos de exigencia pueden interferirse mutuamente al punto de oponerse: "Las personas sujetas tanto a injusticias culturales como económicas necesitan reconocimiento y redistribución. Necesitan afirmar y negar a la vez su especificidad. ¿Cómo sería esto posible, si lo es?” (Fraser, 1997: 26), termina preguntándose. Esta pregunta debe replicarse para nuestro caso: ¿cómo es posible sostener demandas de reconocimiento, derivadas de la situación de los inmigrantes, a través de instrumentos de redistribución socioeconómica, declaradas en las políticas dominadas por el discurso inclusivo?

Esta es precisamente la situación de la inclusión: se halla en un enclave problemático que la sitúa en un dilema; un dilema que supone que para incluir se debe socavar la diferencia entre grupos —en virtud de la exigencia

decir, desde una perspectiva que permite evaluar las prácticas y, en virtud de ello, corregirlas cuando se llevan a cabo incorrectamente. Un caso muy especial es el de Hönneth (2013) quien, en el texto referido da cuenta de los obstáculos para que esta consideración "normativa" no fuera posible en la historia de la filosofía. 
de desdiferenciación distributiva-, al tiempo que promover su identidad - por mor de la exigencia de diferenciación del reconocimiento--

Aquí radica la razón de que las iniciativas inclusivas en el sistema educativo se perciban degradadas en la práctica y, en el extremo, diluidas al punto de considerarse un vacío ideario: igualar y diferenciar al mismo tiempo requiere salir del dilema planteado por Fraser y exige, en consecuencia, hacer un balance que posibilite dilucidar sus efectos sobre la situación de personas y grupos. Una situación, según se ha indicado, en la que la inclusión se ha convertido en el desiderátum de la política educativa, sin que no obstante se perciba si su resultado es siquiera relevante. Pues bien, una adecuada caracterización de tal situación, permitirá ponderar de mejor forma el potencial de las políticas de educación inclusiva.

\section{LA FUERZA NORMATIVA DEL RECONOCIMIENTO}

Las cuestiones de la identidad personal y colectiva, así como la discusión respectiva acerca de la función del reconocimiento en relación con ellas, constituyen un apartado relevante de las explicaciones que se ofrecen actualmente sobre las transformaciones experimentadas por las democracias modernas. Entre ellas, aquellas transformaciones producidas por la ingente cantidad de inmigrantes que transitan entre naciones. De hecho, un motivo que plantea la necesidad de aclarar las relaciones entre identidad y reconocimiento, proviene del debate concerniente a la legitimidad o no de la inclusión de personas pertenecientes a grupos minoritarios en comunidades sociales dominantes o mayoritarias (así como la consiguiente demanda por sus derechos).

Esta consideración no debe ser solo el objeto de una descripción empírica, puesto que la forma en que se trata a los excluidos revela el núcleo normativo de una sociedad. Así lo entiende el sentido común, que acostumbra recordar que la justicia se deja ver nítidamente en la forma en que la sociedad trata a los que fracasan en ella. Este hecho queda revelado, en un sentido que no lo hace el uso de otra clase de expresión, el carácter normativo del uso de "Nosotros" (Brandom, 1994) y, por tanto, pone de manifiesto el carácter normativo de nuestras prácticas:

Las "teorías del reconocimiento" se hacen cargo de este hecho [es decir, del individuo como logro histórico con múltiples raíces y consecuencias] ofreciendo una teoría filosófica - esto es, normativa, aunque con sólidas bases empíricas- de lo que podríamos llamar una intersubjetividad "originaria" -aquella que es expresada en una relación de reconocimiento recíproco en- 
tre dos individuos numéricamente distintos- sólo a partir de la cual es posible una diferenciación cualitativa [...] entre dos agentes racionales. (Ormeño, 2016: 67)

Este es el contexto en que la inmigración, por ejemplo, se ha venido transformando en uno de los principales desafíos de las sociedades occidentales. Y aunque este no es el lugar para entrar en detalles al respecto, la "cuestión migratoria" no es solo un asunto de números, vale decir, del volumen total de los migrantes (Mazza, 2015); es, antes bien, un problema que toca el centro de los principios básicos de comunidades políticas basadas en el respeto de los derechos. En el caso de Chile, la inmigración extranjera constituye, de hecho, un prisma paradigmático, que permite examinar la situación general de grupos socialmente excluidos y que evidencia las múltiples formas de exclusión de que son objeto (Kaufmann, 2011; Muñoz-Pérez, Alarcón-Leiva \& Sanhueza-Henríquez, 2018).

Parte de tal prisma depende de concebir adecuadamente, en primer lugar, las cuestiones de la identidad. Taylor ofrece una aproximación que permite justificar la diferencia entre la identidad personal y la de grupos, aludiendo en el primer caso al sentido psicológico de "la identidad" y a la formación de las identidades colectivas (Taylor, 1996: 14). Respecto al sentido psicológico o individual, Taylor sostiene que pese a la dificultad que presenta ofrecer una definición estricta de tal identidad, "se podría decir que $[. .$.$] define de alguna manera el horizonte de mi mundo moral" (Tay-$ lor, 1996: 10). Ello ocurre en el mismo sentido en que mi "identidad social" me inscribe en un espacio determinado, es decir, en que mi identificación permite ubicar en un campo social; mi identidad "me sitúa en el paisaje moral" (Taylor, 1996: 11).

Sin embargo, el solo eje moral no permite entender del todo las implicaciones de la identidad. La razón del carácter moderno de la identidad personal, se muestra a propósito de la necesidad de haber admitido previamente "la idea de que cada ser humano tiene su propia manera de ser" (Taylor, 1996: 11) y que los asuntos relativos al horizonte último no se plantean únicamente en el registro de lo universal -humano, de clase, de rango, de status-, ante bien recaen sobre los individuos, lo cual lo atribuye Taylor a la "revolución" suscitada por Herder.

Una vez perdida la influencia del rango para el destino social del individuo, y abierto el espacio de la negociación con el entorno y la historia de cada quien, para que a su vez quedara abierta la posibilidad que los seres humanos puedan convertirse en lo que quisieran o en aquello que tuvieran capacidad de ser; entonces, se ha entrado en el contexto de una sociedad (cada vez) más igualitaria.

No obstante, faltaba algo más que la sola "revolución igualitarista": 
Se necesitaba también esta revolución expresivista [...] que reconoce en cada individuo su propio modo de ser humano y que [...] le conjura a realizarlo en toda su originalidad [...] Esto otorga un nuevo sentido, más radical, al ideal de la autenticidad, a la fidelidad hacia uno mismo. (Taylor, 1996: 12)

La participación del individuo en la definición de su identidad resulta fundamental, en la medida en que negocia con su entorno, pero no dispone de su plena voluntad: de ahí que la época de la revolución expresivista sea también la época en que surge otro discurso, el discurso del reconocimiento: "Es el espacio del reconocimiento exigido, pero susceptible de ser rechazado. Este espacio es el que da lugar al discurso y a las teorías del reconocimiento" (Taylor, 1996: 13).

Este segundo eje desemboca en la identidad de grupo. La identidad de grupo es una consecuencia, entonces, del proceso de desarrollo de la identidad individual y realiza, diríase, plenamente la identidad individual. Por ello mismo se ha vuelto un eje tan central en la política moderna (Anderson, 1991). Por identidad de grupo, hemos de entender aquel referente normativo de que habla Brandom cuando explica el uso de la expresión "Nosotros". Es decir, reconocimiento y referencia a un "Nosotros" constituye la base de la fuerza normativa exigida mediante los mecanismos de reconocimiento, tal como los explica Hönneth (1992; 2006).

En términos de Hönneth, la ofensa y el desprecio constituyen la otra cara de la moneda del reconocimiento. ¿Qué entiende Hönneth por "reconocimiento"? En el segundo de los artículos mencionados, define "reconocimiento" diciendo que se trata de "afirmación positiva de sujetos o grupos" y que en un acto de reconocimiento "es ante todo mediante el correspondiente modo de comportamiento como es generada la credibilidad que para el sujeto reconocido es de importancia normativa" (Hönneth, 2006: 134). En consecuencia, para Hönneth, el reconocimiento se expresa en actos concretos, como el amor por el otro o la solidaridad con los otros. Más aún, el amor, el derecho y la solidaridad constituyen "tres patrones del reconocimiento" (Hönneth 1992: 86).

Hay una conexión evidente entre esta noción de reconocimiento y una noción que él desarrolla en el primero de los artículos: la noción de honor o dignidad. "Por "honor", "dignidad" o dicho con un término moderno, por "status" de una persona se entiende la medida de aprecio social que corresponde a su modo de auto realizarse en el horizonte de una tradición cultural de una sociedad" (Hönneth, 1992: 82). Si examinamos estos análisis desde la perspectiva de las minorías, las categorías de "reconocimiento" y de "dignidad humana" debieran considerarse constitutivas. Sin embargo, en ocasiones, en circunstancias eventualmente sistemáticas, las personas somos objeto de malos tratos o de discriminación o, en lenguaje 
de Hönneth, su integridad como persona se ve afectada por el desprecio o por la ofensa (Hönneth, 1992: 82).

Entonces, ¿qué entiende Hönneth por "ofensa" y por "desprecio"? "Ofensa" y "desprecio" son formas de reconocimiento negado. Dichas nociones no representan tanto una injusticia, aunque lo pueden ser evidentemente, que menoscaban "a los sujetos en su libertad o le ocasionan daños", sino que encarnan una conducta dañina por la que las personas son "heridas en su comprensión de sí mismas que han adquirido por vías intersubjetivas" (Hönneth, 1992: 80).

Hönneth reconoce que "ofensa y desprecio" tienen grados distintos que van de la humillación a la degradación de la persona, pasando por la negación de sus derechos fundamentales. En definitiva, la ofensa y el desprecio tienen como consecuencia la pérdida de confianza en sí mismo: quien los padece queda "estructuralmente excluido de la posesión de determinados derechos dentro de una sociedad." (Hönneth, 1992: 81) Tanto es así, que quien es víctima del desprecio o de la ofensa, puede decirse que sufre algún tipo de "muerte psíquica", "muerte social" o alguna forma de "ultraje" (Hönneth, 1992: 82).

¿Puede el reconocimiento tener consecuencias negativas? o, dicho en términos de Hönneth, ¿puede el reconocimiento transformarse en ideología? La respuesta es que el peligro existe, por lo que propone distinguir entre formas del reconocimiento ideológicas y las moralmente exigidas de forma legítima, que hemos descrito con anterioridad.

Una de las características del reconocimiento puramente aparente o falso, "ideológico" lo llama Hönneth, es que reconocer a alguien puede llegar a significar "inducirlo, en virtud de requerimientos repetidos y continuados de forma ritualizada, exactamente al tipo de auto comprensión que encaja adecuadamente en el sistema establecido de expectativas de comportamiento" (Hönneth, 2006: 130). Así, Hönneth quiere alertar acerca de ciertas formas de reconocimiento que pueden transformarse en formas de sumisión encubiertas:

El reconocimiento social puede siempre tener la función de actuar como ideología generadora de conformidad: la repetición continuada de las mismas fórmulas de reconocimiento alcanza sin represión el objetivo de producir un tipo de autoestima que provee de las fuentes motivacionales para formas de sumisión voluntarias. (Hönneth, 2006: 131)

A juicio de Hönneth hay que mostrar el núcleo irracional de las formas ideológicas de reconocimiento, por cuanto es posible que bajo sus máscaras se encubra una práctica de exclusión. En la medida en que se justifica decir que el reconocimiento ideológico es irracional, convendrá subrayar 
el aspecto positivo de esta observación: rectamente entendido, el reconocimiento constituye una forma racional de interacción entre personas.

En este sentido, es necesario precisar por qué es racional y la manera en que lo es, por cuanto creemos que hay una relación (interna) entre la racionalidad del reconocimiento y el carácter normativo de las prácticas sociales, en el sentido que antes lo indicamos. De forma que hay un camino justo que recorrer desde la situación concreta de la díada inclusión/exclusión hasta su explicación teórica, por medio de la exigencia de la racionalidad normativa de las prácticas sociales, que no es otra que la de la justicia.

\section{LA REFERENCIA A UNA TEORÍA DE LA JUSTICIA}

Es bien conocido que entre las contemporáneas teorías de la justicia se viene produciendo un desarrollo cada vez más profundo y amplio. Así como un debate cada vez más intenso y revitalizador de los presupuestos básicos de los enfoques en juego. No cabe proponerse establecer entre tales desarrollos alguna clase de continuidad, por cierto, ni menos esperar un desenvolvimiento progresivo de sus tentativas por abordar más comprensivamente su objeto. Lo que sí corresponde es distinguir las formas teóricas más apropiadas para resolver ciertos problemas, como ocurre con el planteamiento que se ha estado haciendo en relación con la inclusión de inmigrantes en el marco de la política educativa nacional chilena.

Para el caso presente, debemos hacer una descripción de los enfoques con la finalidad de destacar que la justicia entendida como reconocimiento sintetiza, al mismo tiempo que problematiza, las ventajas y desventajas de las dos restantes concepciones, al conectar el enfoque económico de los "bienes primarios" y el enfoque "político" de la "participación", con la dimensión "cultural" de la experiencia de que son objeto grupos socialmente marginados, cuya identificación deriva de la aplicación de diversos criterios de subordinación: raza, etnia, clase y género. Por otra parte, se defenderá que la teoría de la justicia ligada a las políticas del reconocimiento, constituye un mejor referente para juzgar las pretensiones de las políticas inclusivas cuando éstas se refieren a inmigrantes, pues contienen un potencial normativo.

Las aludidas concepciones de justicia social son justicia como distribución (Rawls, 1971), como reconocimiento (Fraser \& Hönneth, 2003) y como participación (Young, 1990; Fraser \& Hönneth, 2003), siendo esta última una suerte de derivado del enfoque por reconocimiento. La primera está centrada en la distribución de bienes, recursos materiales y culturales, y capacidades; la segunda, en el reconocimiento y el respeto cultural de 
todas y cada una de las personas, en la existencia de relaciones justas; mientras que la tercera está referida a la participación.

Importa señalar que la teoría de la justicia que explica mejor la tensión esencial a la que queda sujeta la situación de los inmigrantes - a propósito de las políticas inclusivas, en el marco de referencia del dilema redistribución y reconocimiento-, es la teoría del reconocimiento. La razón de esta elección depende de lo que se ha adelantado ya acerca de la teoría del reconocimiento misma y, además, por las consideraciones que a continuación se detallan.

La teoría del reconocimiento expresa lo que se ha llamado "justicia relacional" o "cultural", definida como ausencia de dominación, de no reconocimiento e irrespeto (Fraser, 1997; Fraser \& Hönneth, 2003). Como señala Fraser (1997), las reivindicaciones de justicia social en el mundo actual parecen dividirse en dos tipos cada vez más claros. El primero, quizás el más afamado, hace referencia a la redistribución, reivindicando precisamente una distribución más justa de bienes y recursos. Hoy en día, sin embargo, es posible encontrarse con un segundo tipo de reivindicaciones en las llamadas "políticas de reconocimiento", que son las que ponen en evidencia la necesidad de disolver la tensión a favor del reconocimiento cultural.

La valoración de las minorías sociales, de género y raciales intenta desarrollar un nuevo paradigma de la justicia que sitúa al reconocimiento en su centro. El ascenso de la política de identidad, ha cambiado el foco de las reivindicaciones de la redistribución igualitaria, pues exige una atención preferente de la diversidad. El distanciamiento generalizado de la política cultural respecto de la política social y de la política de la diferencia respecto a la política de la igualdad, ha llevado a una polarización. Sin embargo, Fraser sostiene que tales antítesis son falsas, puesto que se exige tanto la redistribución como el reconocimiento. Hay que integrar los aspectos emancipadores de las dos problemáticas. La tarea consiste en idear una orientación política programática que integre lo mejor de la política de la redistribución y lo mejor de la política del reconocimiento. Las diferencias entre esta concepción de justicia como reconocimiento y el enfoque de justicia distributiva son resumidas por Fraser (1997) en cuatro aspectos.

En primer lugar, los dos enfoques asumen concepciones diferentes respecto de la fuente de la injusticia: mientras que la justicia distributiva considera a la estructura económica de la sociedad (marginación, explotación y privación), la teoría del reconocimiento supone que las injusticias culturales están vinculadas a procesos de representación, interpretación y comunicación. La primera sostiene, entonces, una concepción material de la injusticia, en tanto que la segunda considera que la principal fuente de injusticias es de raíz simbólica. 
En segundo lugar, ambos enfoques proponen diferentes tipos de soluciones en relación con la injusticia. En el enfoque de la redistribución, el remedio de la injusticia es algún tipo de reestructuración económica; en tanto, en el enfoque del reconocimiento, la solución es el cambio cultural o simbólico o la reevaluación ascendente de las identidades no respetadas o de sus productos culturales. De forma que el modo en que puede subsanarse la situación de injusticia eventualmente identificada, desde una perspectiva y otra difiere en cuanto a que, en la lógica del argumento general aquí defendido, las políticas inclusivas requieren soluciones culturales, simbólicas o de reevaluación ascendente y no, como de hecho tiene a ocurrir en la implementación de tales políticas inclusivas en educación, una respuesta de base material; es decir, incrementando el monto de la subvención por estudiante.

En tercer lugar, los dos enfoques asumen concepciones diferentes de las colectividades que sufren la injusticia. En el enfoque de la redistribución, los sujetos colectivos de injusticias son clases o colectividades definidas por el mercado o por los medios de distribución; en el enfoque del reconocimiento, en cambio, se plantea la necesidad de reconocimiento en relación con la raza, la sexualidad, etc. Primordialmente, esta es la razón de que las políticas inclusivas de carácter "focalizado", desplegadas por los diagnósticos institucionales fallen en sus resultados. La "focalización de la inversión pública" en grupos "vulnerables", "marginados", "de bajos ingresos", renuncia de entrada a la pretensión universalistas contenida en toda política qua inclusiva. Abandonado su punto de partida universalista, todo lo que sigue es una profundización de las desigualdades de origen.

En cuarto lugar, finalmente, las diferencias de grupo se consideran desde el primer enfoque como diferenciales de injusticia, ligadas a estructuras socialmente injustas $y$, por tanto, tales estructuras se deben abolir. Por el contrario, en el enfoque del reconocimiento los diferenciales de injusticia están vinculados a una jerarquía de valores y requieren re-evaluarse los rasgos devaluados. En este último sentido, el paso de la situación de injusticia original a una nueva situación de justicia, requiere cambio cultural, no un mero ajuste de cuentas económico; a despecho que en ocasiones este sea también el caso. Lo que se pretende subrayar al formular de esta manera dicotómica el punto es su claridad, sin perder de vista, no obstante, que usualmente, como Fraser y Butler (Butler \& Fraser, 2016) han señalado a su debido tiempo, aunque defendiendo posiciones parcialmente opuestas, la falta de reconocimiento es también falta de recursos para solventar los requerimientos materiales de la subsistencia.

En este sentido, Hönneth (Fraser \& Hönneth, 2003) considera que una de las formas de falta de respeto o minusvaloración de las personas 
está directamente relacionada con la participación democrática. De manera que los ciudadanos sin participación democrática, se encuentran estructuralmente excluidos de determinados derechos. El hecho de experimentar la negación de estos derechos conlleva falta de consideración propia y de respeto hacia uno mismo. Así, existiría un vínculo entre ausencia de respeto y reconocimiento y falta de participación en la comunidad.

Esta conexión es evidente para Iris Marion Young (1990), quien sostiene que el enfoque de justicia social necesita una perspectiva más extensa acerca de la eliminación de la opresión y dominación institucional. Por lo pronto, la mera solución técnica de la economía de la redistribución de recursos materiales parece ser insuficiente, aunque necesaria. Conviene avanzar hacia un enfoque sobre justicia centrado en procesos políticos. Ello incluye tanto la injusta distribución de bienes materiales como la injusta distribución del reconocimiento social y, en consecuencia, da lugar a una concepción de la justicia basada en una racionalidad ampliada.

Circunscribir la situación de los inmigrantes en el sistema escolar, y la situación de cualquier minoría en cualquiera de los subsistemas sociales, a la dotación de recursos para cubrir sus necesidades, es una tendencia ostensiblemente errada. Se trata, más bien, de ofrecer como base reconocimiento a su diferencia para así lograr una mayor integración a la comunidad de destino. Este reconocimiento no puede, no obstante, provenir de un vacío sentido de inclusión. Un sentido que no fuerza ni conmina a los agentes a actuar en consonancia con la necesidad de brindar a los inmigrantes un horizonte de integración social pleno y un reconocimiento también pleno a su dignidad. Un tal horizonte sólo puede surgir del hecho de mostrar que el mutuo reconocimiento es una demanda normativa que es parte de la arquitectura de toda relación entre seres humanos.

En el marco de esta convición, y comentando lo que el sociólogo F. Dubet llama la "norma de la igualdad" y la "norma del rendimiento" en el sistema educativo, Hönneth afirma en una entrevista:

Esto me parece una idea altamente fructífera, que naturalmente se podría traducir con facilidad a mi teoría de reconocimiento: nuestro sistema escolar se dirige a las alumnas y los alumnos o bien como a futuros ciudadanos, con la pretensión de que tengan las mismas posibilidades de participación y colaboración, o bien como a portadores de rendimientos en el mercado de trabajo, con todas las diferencias ya existentes en el correspondiente perfil del rendimiento [...] Sobre cuál de los dos principios de reconocimiento domina en la escuela en cada momento, deciden siempre, tal como lo veo yo, las disputas político-morales dentro del ámbito público acerca del papel y de la tarea de la educación escolar; y, según mi impresión, no hay duda de que hoy en día, en la ejecución de la llamada trasformación "neoliberal" del 
capitalismo occidental, el segundo principio de reconocimiento ha alcanzado el predominio [...]. (Hernàndez I Dobon, Herzog \& Martins, 2017: 403)

En esta glosa, Hönneth pone el acento en la necesidad que el sistema educativo apoye la formación de ciudadanos, comprometidos con un sentido de comunidad que, no obstante, parece estar lejos de las motivaciones que animan al sistema educativo chileno. Siendo este el caso, no obstante, parece evidente que un sistema escolar debe estar basado en el principio de reconocimiento. La prioridad de este principio de reconocimiento, por otra parte, hace suponer que una teoría del reconocimiento tendría mucho que aportar a la superación de la oposición entre socialización e individuación que ha caracterizado el desarrollo educativo.

No puede seguir ocurriendo que en nuestro sistema educativo se imponga la idea de que la escuela en todos sus niveles y modalidades sea no más que un sistema performativo, un sistema en el que la exigencia del rendimiento conduzca a grado cero las necesidades de la realización personal y colectiva. La recuperación del ideal normativo de un "Nosotros" es la base de esta orientación, sin la cual no podrá salirse del modelo de la competencia que supone como para tarea de la escuela tener que decidir o socializar o individuar, sin resolver nunca su oposición. Un sentido integral, digamos, que no es mera retórica, sino humanización.

\section{REFERENCIAS}

Ahmed, S. (2007). The language of diversity. Ethnic and Racial Studies, 30(2), 235256.

Ainscow, M. \& Miles, S. (2008). Por una educación para todos que sea inclusiva. ¿Hacia dónde vamos ahora? Perspectivas, 38(1), 17-44.

Anderson, B. (1991). Imagined comunities. London: Verso.

Armijo-Cabrera, M. (2018). Deconstruyendo la noción de inclusión: Un análisis de investigaciones, políticas y prácticas en educación. Revista Electrónica Educare (Educare Electronic Journal), 22(3), 1-26.

Bauman, Z. (2005). Identidad. Buenos Aires: Losada.

Berlant, L. (2011). Cruel Optimism. Durham and London: Duke University Press.

Blanco, R. (2006). La equidad y la inclusión social: Uno de los desafíos de la educación y la escuela hoy. REICE - Revista Electrónica Iberoamericana sobre Calidad, Eficacia y Cambio en Educación, 4(3), 1-15.

Booth, T. \& Ainscow, M. (2002). Guía para la evaluación y mejora de la educación inclusiva. Desarrollando el aprendizaje y la participación en las escuelas. Madrid: Consorcio Universitario para la Educación Inclusiva.

Brandom, R. (1994). Making it Explicit. Cambridge Mass.: Harvard University Press. 
Butler, J. \& Fraser, N. (2016). ¿Redistribución o Reconocimiento? Un debate entre marxismo y feminismo. Madrid: Traficantes de Sueños.

Cierani, P., García, L. \& Gómez, A. (2014). Niñez y adolescencia en el contexto de la migración: principios, avances y desafíos en la protección de sus derechos en América Latina y el Caribe. REMHU - Rev. Interdiscipl. Mobil. Hum., (42), 9-28.

Fraser, N. (1997). Justice interruptus. Critical reflections on the "Postsocialist" Condition. New York: Routledge.

Fraser, N. \& Hönneth, A. (2003). Redistribution or recognition? A political-philosophical exchange. London: Verso Press.

Galaz, G., Poblete, R. \& Frías, C. (2017). Políticas Públicas e Inmigración ¿Posibilidades de Inclusión efectiva en Chile? Santiago: Editorial Universitaria.

Graham, L. J. \& Slee, R. (2008). An illusory interiority: Interrogating the discourse/s of inclusion. Educational Philosophy and Theory, 40(2), 277-293.

Gutman, A. (1987). A Democratic Education. Priceton, N. J.: Princeton University Press.

Hernàndez I Dobon, F. J., Herzog, B. \& Martins, M. R. (2017). La Educación y la Teoría del Reconocimiento: entrevista a Axel Honneth. Educação \& Realidade, 42(1), 395-406.

Hönneth, A. (1992). Integridad y desprecio. Motivos básicos de una concepción de la moral desde la teoría del reconocimiento. Isegoría, (5), 78-92.

Hönneth, A. (2006). El reconocimiento como ideología. Isegoría, (35), 129-150.

Hönneth, A. (2013). La educación y el espacio público democrático. Un capítulo descuidado en la Filosofa Política. Isegoría, (49), 377-395.

Infante, M. \& Matus, C. (2009). Policies and practices of diversity: remaining possibilities for new discourses. Desability \& Society, 24(4), 237-445.

Informe OBIMID (2016). La migración en Chile: breve reporte y caracterización. Disponible en https://www.researchgate.net/publication/305618995_La_Migracion_en_Chile_Breve_reporte_y_caracterizacion

Kaufmann, S. (2011). Multiculturalidad y ética del reconocimiento. Revista Persona y Sociedad, 25(1), 75-87.

Knight, T. (1999). Inclusive education and educational theory: Inclusive for what? Paper presented at the British Educational Research Association Conference. Brighton: University of Sussex. Disponible en http:/ / www.leeds.ac.uk/edu$\mathrm{col} /$ documents $/ 000001106 . \mathrm{htm}$

Lindblad, S. \& Popkewitz, T. (Eds.) (2001). Education governance and social integration and exclusion: Studies in the powers of reason and the reasons of power (Uppsala Reports on Education, 39). Uppsala: Uppsala University. Disponible en https://www.gu.se/digitalAssets/1320/1320558_finalreport-egsie2.pdf

Madero, I. (2011). Inclusión y exclusión de género y clase al interior de la escuela chilena en 4 comunas del sur de Chile. Estudios Pedagógicos, 37(2), 135-145.

Matus, C. \& Infante, M. (2011) Undoing diversity: knowledge and neoliberal discourses in colleges of education. Discourse: Studies in the Cultural Politics of Education, 32(3), 293-307. 
Mazza, D. O. (2015). Direito humano a mobilidades: dois textos e dois contextos. Revista Interdisciplinar de Mobilidade Humana, 23(44), 237-257.

Ministerio de Educación de Chile (MINEDUC) (2009a). Ley Núm. 20.370/2009. Establece Ley General de Educación. Santiago: Biblioteca del Congreso Nacional de Chile. Disponible en http://bcn.cl/1uvx5

Ministerio de Educación de Chile (MINEDUC) (2009b). Decreto N 170/2009. Fija normas para determinar los alumnos con necesidades educativas especiales que serán beneficiarios de las subvenciones para educación especial. Santiago: Biblioteca del Congreso Nacional. Disponible en http://www.leychile.cl/Navegar?idNorma $=1012570$

Ministerio de Educación de Chile (MINEDUC) (2015a). Ley Núm. 20.845/2015. De inclusión escolar que regula la admisión de los y las estudiantes, elimina el financiamiento compartido y prohíbe el lucro en establecimientos educacionales que reciben aportes del Estado. Santiago: Biblioteca del Congreso Nacional de Chile. Disponible en http://www.leychile.cl/Navegar?idNorma $=1078172$

Ministerio de Educación de Chile (MINEDUC) (2015b). Decreto N83/2015. Aprueba criterios y orientaciones de adecuación curricular para estudiantes con necesidades educativas especiales de educación parvularia y educación básica. Disponible en http://especial.mineduc.cl/wp-content/uploads/sites/31/2016/08/Decreto-83-2015.pdf

Ministerio de Educación de Chile (MINEDUC) (2018a). Mapa del estudiantado extranjero en el sistema escolar chileno (2015-2017). Documento de Trabajo No 12. Santiago de Chile. Disponible en https://www.mineduc.cl/wp-content/uploads/sites/19/2018/05/MAPA_ESTUDIANTES_EXTRANJEROS_SISTEMA_ESCOLAR_CHILENO_2015_2017.pdf

Ministerio de Educación de Chile (MINEDUC) (2018b). Política Nacional de Estudiantes Extranjeros 2018-2022. Disponible en https://migrantes.mineduc.cl/wp-content/uploads/sites/88/2018/06/POLITICA-NACIO-

NAL-EE-Final-1-1.pdf

Muñoz-Pérez, E., Alarcón-Leiva, J. \& Sanhueza-Henríquez, S. (2018). Modernidad, tolerancia y migración: consecuencias para la educación en Chile. Educ. Soc., Campinas, 39(144), 756-778.

Ormeño, J. (2016). Las eventuales consecuencias políticas que implicaría la adopción del "Reconocimiento" como paradigma de la teoría social. Perspectiva Filosófica, 43(2), 67-90.

Organización Internacional para las Migraciones (OIM) (2018). Dinámicas migratorias en fronteras de países de América del Sur. Cuaderno $\mathrm{N}^{\circ} 10$. Disponible en https://robuenosaires.iom.int/sites/default/files/publicaciones/Cuaderno_10-Dinamicas_Migratorias_en_Fronteras_de_paises_de_America_del_Sur.pdf

Pavez, I. (2017). La niñez en las migraciones globales: perspectivas teóricas para analizar su participación. Tla-Melaua, Revista de Ciencias Sociales, (41), 96-113. 
Pelletier, C. (2011). Beating the barrel of inclusion: Cosmopolitanism through Rabelais and Rancière - A response to John Adlam and Chris Scanlon. Psychodynamic Practice, 17(3), 255-272.

Rambla, X. et al. (2008). La educación inclusiva frente a las desigualdades sociales. Perspectivas, 38(1), 81-96.

Rawls, J. (1971). A theory of justice. Cambridge, MA: Harvard University Press [Trad. al español (1978). Teoría de la Justicia. México: FCE].

Rothon, C. (2007). Can achievement differentials be explained by social class alone? An examination of minority ethnic educational performance in England and Wales at the end of compulsory schooling. Ethnicities, 7(3), 306-322.

Rothon, C. (2008). Women, men and social class revisited: An assessment of the utility of a 'combined' schema in the context of minority ethnic educational achievement in Britain. Sociology, 42(4), 691-708.

Sen, A. (2009). The idea of Justice. Nueva York: Penguin Press.

Taylor, C. (1996). Identidad y Reconocimiento. Revista Internacional de Filosofía Politica, (7), 10-19.

Tomasevsky, K. (2004). El asalto a la educación. Buenos Aires: Universidad de Buenos Aires.

Tomlinson, S. (1982). A sociology of special education. London: Routledge and Kegan Paul.

UNESCO (2015). Éducation 2030. Déclaration d'Incheon. Vers une éducation de qualité inclusive et équitable et un apprentissage tout au long de la vie pour tous. En Forum mondial sur l'éducation 2015. Incheon, République de Corée. Disponible en http://unesdoc.unesco.org/images/0024/002456/245656f.pdf

Walzer, M. (1997). Las esferas de la justicia. México D. F.: Fondo de Cultura Económica.

Young, I. M. (1990). Justice and the politics of difference. New Jersey: Princeton University. 